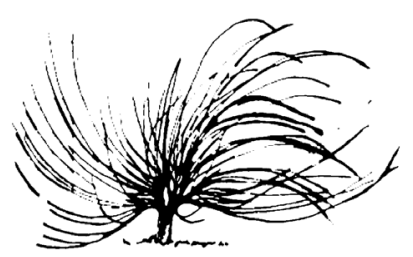

\title{
El personal docente universitario en el siglo XXI: Retos desde la educación superior pública costarricense
}

\author{
Yarina Paniagua-Cortés ${ }^{1}$ \\ Hefei Ivy Experimental Academy \\ Costa Rica \\ yarinapaniagua@gmail.com
}

\begin{abstract}
Resumen
El perfil del personal docente universitario que se ha trabajado por muchos años ya no responde a las necesidades del mundo en el que vivimos. Tener experticia en su área disciplinar, tener amplia experiencia profesional y producir conocimiento se aleja de ser suficiente para enfrentar las complejidades de nuestra sociedad actual. En este ensayo, se discuten diferentes cualidades que, además de las anteriores, son indispensables para el personal docente universitario en el siglo XXI, tales como: experticia pedagógica, manejo de competencias emocionales, individualización del aprendizaje, dominio de herramientas tecnológicas, redes académicas, movilidad laboral, dominio de una segunda lengua, mejoramiento continuo y reflexión sobre su propia praxis para que su perfil esté en concordancia con los exigencias de la sociedad y las demandas del estudiantado, de manera que su labor sea pertinente en la actualidad y como preparación para el futuro.
\end{abstract}

Palabras clave: Educación, docencia, enseñanza superior, perfil profesional, evaluación del personal docente.

http://dx.doi.org/10.15359/rep.esp-21.4

1 Docente de inglés, lengua y literatura. Máster en Educación con énfasis en Aprendizaje del Inglés y Máster en Educación con énfasis en Docencia Universitaria, ambas de la Universidad Nacional. 


\begin{abstract}
The hiring profile for professors that has been widely accepted for years in higher education institutions does not guarantee that these professionals develop all the skills required to fulfill the needs the world has today. In most cases, this profile includes being an expert in their area, having relevant professional experience, and doing research to produce knowledge and new advances. However, this is no longer sufficient, considering how complex and constantly changing our world is. That is why this essay discusses other qualities that are essential to any higher education professor in our current society. Pedagogical expertise, emotional competence, differentiation, knowledge of technological tools and their implications, being part of academic networks, mobility, foreign language proficiency, professional development and reflection are essential in order to fulfill both society's and students' needs so that professors' work is pertinent now and as preparation for the future.
\end{abstract}

Keywords: education, teaching profession, higher education, professional profile, teacher evaluation.

\title{
Introducción
}

$\mathrm{E}$ 1 docente universitario tiene una labor compleja, que implica trabajar desde múltiples aristas para que se pueda ejercer la profesión con propiedad, por ejemplo, la pedagogía, la investigación, la cognición, las emociones y la motivación, entre otras. Por mucho tiempo, las instituciones de educación superior han buscado contratar educadores con experticia en su área disciplinar y con amplia experiencia en su campo. También se ha dado gran valor al hecho de que el profesorado universitario produzca, mediante investigaciones, nuevo conocimiento, lo que es un requisito en las comunidades científicas hoy en día.

Las universidades estatales costarricenses son ejemplos de la búsqueda de las cualidades mencionadas anteriormente en profesionales de la educación superior. Estas instituciones cuentan con requisitos de contratación y ascenso en sus respectivos regímenes de carrera académica, los cuales consideran aspectos como el grado académico, la experiencia y las publicaciones. 
Todas las universidades estatales comparten los criterios de calificaciones profesionales; en términos del grado académico, el mínimo requerido para la contratación docente en la Universidad Técnica Nacional (2017), en la Universidad Estatal a Distancia (2018a) y en el Tecnológico de Costa Rica (2018a) es el bachillerato universitario o, de no contar con el nivel de bachillerato, las dos primeras universidades solicitan un certificado de idoneidad (120 créditos aprobados). Las instituciones que tienen como requisito un grado mínimo de maestría o especialidad de posgrado superior a la licenciatura son la Universidad de Costa Rica (2008) y la Universidad Nacional (2018).

Con respecto a la experiencia laboral, las únicas universidades públicas que la consideran un requisito para la contratación de docentes son el Tecnológico de Costa Rica, que demanda un mínimo de uno a tres años (2018a), y la Universidad Nacional (2018), que a pesar de que lo estipula en su normativa no tiene un tiempo mínimo definido.

Además de los requisitos de contratación, en los regímenes de carrera académica, existen factores a tomar en cuenta para ascender que reflejan también la importancia de la producción académica, experiencia laboral y experticia en el área disciplinar para las instituciones públicas de educación superior en Costa Rica. El dominio de lenguas extranjeras es considerado por cuatro de las cinco instituciones de educación superior públicas, la Universidad Técnica Nacional es la excepción (Universidad Estatal a Distancia, 2018b; Tecnológico de Costa Rica, 2018b; Universidad de Costa Rica, 2008; Universidad Nacional, 2015). Por su parte, la producción académica es un requisito para ascender en los regímenes de carrera académica de todas las universidades públicas del país, sin embargo, en la Universidad Técnica Nacional, el personal docente interino no forma parte de dicho régimen. Por último, la evaluación docente es tomada en cuenta para ascender únicamente en la Universidad Nacional (2015), la Universidad de Costa Rica (2008) y el Tecnológico de Costa Rica (2018b).

En la coyuntura actual, a pesar de la incuestionable importancia que dichos requisitos tienen, estos distan de ser suficientes para contar con grupos profesionales idóneos, que realmente sobresalgan en su labor y se puedan enfrentar, sin problemas, a las necesidades de una sociedad que se encuentra en constante cambio. Partiendo del hecho de que las universidades son instituciones que pretenden destacarse por su excelencia académica, innovación, producción científica y acción 
social pertinentes, tanto a nivel nacional como internacional, y siempre en concordancia con las necesidades actuales, es sencillo afirmar que los requisitos de contratación, mencionados anteriormente, están lejos de cumplir con lo necesario para lograr este objetivo.

Tal como lo afirma Zabalza (2009),

No es fácil ser profesor/a universitario en la actualidad. Se abren muchos caminos y triunfa la policromía en los guiones de actuación. Estamos, definitivamente, en otra universidad. Y cabe deducir que tendremos que acomodarnos a un nuevo perfil de docentes. En eso estamos, y con toda la perplejidad y desazón que cualquier cambio de papeles trae consigo. (p. 69)

Resulta fundamental, entonces, definir este nuevo perfil de docentes que menciona Zabalza (2009), que es el requerido para la praxis pedagógica universitaria en la actualidad.

Este ensayo, considerando la obligatoriedad histórica de la experticia disciplinar, experiencia profesional y producción académica, aborda otras características indispensables para una educación superior de calidad. El personal docente universitario del siglo XXI debe tener experticia pedagógica, manejo de competencias emocionales y dominio de herramientas tecnológicas, así como formar redes académicas o pertenecer a ellas, dominar al menos un segundo idioma, procurar un aprendizaje individualizado y su propio mejoramiento continuo para que pueda atender las demandas de la población estudiantil en una sociedad en constante transformación.

\section{El perfil del personal docente universitario}

Para poder realizar su labor de manera apropiada, es imperativo que cada profesional tenga un alto nivel de formación en pedagogía. Es decir, que conozca a profundidad áreas como currículo, evaluación y teoría relacionada con el aprendizaje y los procesos cognitivos, de manera que los pueda utilizar en su clase tomando en cuenta las características de cada estudiante. Ejercer la docencia sin tener experticia pedagógica alguna es tan común como inapropiado. Y es que el quehacer fundamental del personal docente universitario es precisamente ese: la docencia. 
Un personal docente que no esté capacitado para educar, aunque sea erudito en su área de especialidad, no puede ofrecer una buena formación a sus estudiantes, lo que se opone a la función sustantiva de la universidad. Es así como lo plantea Zabalza (2002), cuando menciona:

La facilidad de acceso [a la información] no supone una ayuda significativa en lo que se refiere ala [sic] decodificación, asimilación y aprovechamiento de esa información, ni garantiza la vinculación de dicho material con la práctica profesional. Es justamente en esta función de «aprendizaje» (la más sustantivamente «Informativa») donde el profesorado universitario debe centrar su acción. (p. 7)

En presencia de este vacío, las universidades estatales costarricenses tienen como requisito que sus cuadros académicos reciban capacitación en pedagogía una vez contratados. La Universidad Nacional (2018), la Universidad de Costa Rica (2008), la Universidad Estatal a Distancia (2018a) y la Universidad Técnica Nacional (2017) otorgan un plazo de 12 a 18 meses para que sus docentes lleven únicamente un curso de didáctica universitaria o pedagogía universitaria.

Por su parte, el Tecnológico de Costa Rica establece que es responsabilidad del profesorado "dominar los principios educativos que fundamentan su labor docente" y "actualizarse en el campo educativo y en el de su especialidad de conformidad con las políticas institucionalmente establecidas" (2008a, Capítulo 4, párr. 9-10), mas no se observa en la normativa consultada acción alguna al respecto. No obstante, a pesar del esfuerzo de dichas instituciones por ofrecer formación básica en pedagogía, esta es precisamente eso: básica. Por ello no se puede comparar con poseer la experticia en el área, que es lo que se requiere para desempeñar la docencia con propiedad y así dar al estudiantado, centro del acto educativo, una formación de calidad.

La experticia pedagógica es indispensable en la labor educativa universitaria, ya que debe desarrollar tanto los aprendizajes académicos en sus estudiantes como las otras habilidades para su convivencia en la sociedad, que no necesariamente van relacionadas con la adquisición de conocimientos, tales como: el pensamiento crítico, la formación en valores, "la comunicación, la alfabetización digital, la resolución de 
problemas, el trabajo en equipo" (UNESCO, 2015b, p. 64); es decir, una enseñanza que busque educar al ser humano de manera integral.

Parte de estas habilidades no académicas e imprescindibles para una formación integral son las competencias emocionales. Bisquerra (2016) plantea dichas competencias "con la intención de contribuir a dar respuesta a las necesidades sociales que no quedan suficientemente atendidas en las materias académicas ordinarias" (p. 20). Esta carencia en la educación se ha extendido por varios años, pero ya no podemos continuar cometiendo el mismo error. Conocerse a sí, manejar las emociones propias y desarrollar empatía por las ajenas, especialmente en situaciones de conflicto, son destrezas que la humanidad requiere cada vez más. Esta necesidad se ve reflejada en los ámbitos laboral, docente, estudiantil y personal.

En los entornos laborales, la inteligencia emocional hace la diferencia, a tal punto que solo quienes la tienen más desarrollada se convierten en verdaderos líderes. Así lo afirma Goleman (2015):

No es que el CI y las capacidades técnicas sean irrelevantes. Importan, pero principalmente como «competencias de umbral», es decir, son requerimientos de acceso a los puestos ejecutivos. Pero mi investigación, junto a otros estudios recientes, sugiere que la inteligencia emocional es el sine qua non del liderazgo. Sin ella, una persona puede tener el mejor entrenamiento del mundo, una mente incisiva y analítica, y una provisión interminable de buenas ideas, pero aun así no será un gran líder. (p. 12)

Otro ámbito en el que se evidencia la necesidad de desarrollar competencias emocionales es la docencia. Diversos autores visibilizan que la realidad laboral docente está llena de desafíos emocionales y existe un vacío, en términos de educación emocional, que ya tiene consecuencias en los grupos profesionales en esta área. Esteras et al. (2016) expresan que "las investigaciones están confirmando la aparición del síndrome de burnout como una grave amenaza que se está generalizando en los profesionales de la educación" y definen este padecimiento como "un estado psicológico de agotamiento, cansancio emocional y despersonalización" (p. 1178).

Este síndrome de agotamiento se debe, entre otras cosas, a que el aula es un espacio donde comparten desde dos hasta más de cuarenta 
personas; todas ellas con bagajes, necesidades y aspiraciones distintas, incluyendo a cada docente. Ahí surgen acontecimientos que detonan diversas emociones en estudiantes y docentes, y que pueden generar conflictos. Por eso, se hace necesario el manejo de competencias emocionales tanto en docentes como en estudiantes.

Mérida y Extremera (2017) coinciden con este planteamiento y manifiestan que la docencia es emocionalmente demandante:

Una gran parte de las actividades profesionales de los docentes, en la medida en que son actuaciones interpersonales, precisan de la puesta en marcha de estrategias para la percepción, la comprensión y el manejo de los estados afectivos. Además, podría [sic] ayudar al profesorado a afrontar situaciones emocionalmente delicadas en su quehacer diario. (p. 375)

Además de las áreas docente y profesional, la educación emocional es indispensable a nivel personal, y es aquí en donde se vuelve necesario que el personal docente universitario, además de desarrollar competencias emocionales en su propia persona, las desarrollen también en sus estudiantes. Los seres humanos, sin excepción, experimentamos emociones negativas como el miedo, ansiedad, estrés, tristeza e ira. Todas estas emociones son causa de malestar e incluso de trastornos psicológicos, sobre todo, si no se manejan en forma apropiada. Por ejemplo, la ira es la base de todo tipo de violencia, mientras que la tristeza puede provocar depresión (especialmente en procesos de duelo). Según Bisquerra (2011, p.12), "aprender a tomar conciencia de estas emociones para regularlas de forma apropiada puede contribuir a prevenir trastornos emocionales y potenciar el bienestar".

Este autor también afirma:

Las emociones negativas son inevitables. ... En cambio las emociones positivas hay que buscarlas. Y aun así a veces no se encuentran. Por esto, tal vez, sea mejor aprender a construirlas. ... Conviene tener presente que la construcción del bienestar incluye la regulación de las emociones negativas y la potenciación de las positivas. (2011, p. 13) 
Esto es, precisamente, lo que las competencias emocionales ayudan a desarrollar.

Bisquerra (2016) plantea la existencia de cinco competencias emocionales. La primera de ellas es la conciencia emocional, que significa reconocer las emociones propias y ajenas y saber qué comportamientos o situaciones provocan esas emociones en nosotros o nosotras. Esta viene de la mano con la regulación emocional, que significa manejar y expresar las emociones de forma apropiada, incluyendo la autorregulación, así como tener buenas estrategias de afrontamiento y la capacidad para autogenerarse emociones positivas. La tercera competencia es la autonomía emocional y se relaciona con la autoestima, la responsabilidad, la actitud positiva ante la vida, la resiliencia y la capacidad para buscar ayuda cuando se necesita.

Además, este autor propone la competencia social, que está vinculada con la comunicación asertiva y efectiva, el altruismo, la prevención y gestión de conflictos, el liderazgo emocional, el trabajo en equipo y el dominio de habilidades sociales básicas como saludar, despedirse, agradecer, entre otras. Finalmente presenta las habilidades de vida para el bienestar, que incluyen la capacidad de adoptar comportamientos adecuados para enfrentar los desafíos de la vida, el poder organizar nuestra vida de manera sana y equilibrada y el propiciarse para sí de experiencias de satisfacción y bienestar (Bisquerra, 2016).

Es claro que la sociedad requiere personas y profesionales con competencias emocionales. Por ende, es apenas lógico pensar que quienes se encargan de la educación y formación de estas personas deben desarrollar dichas competencias también. Tal como lo expresa Bisquerra (2016):

El primer destinatario de la educación emocional es el profesorado. En primer lugar, porque necesita las competencias emocionales para el ejercicio de su profesión (...) En segundo lugar, para poder contribuir al desarrollo de las competencias emocionales en su alumnado. (p. 19)

Estas cinco competencias son de suma importancia en el ambiente educativo, ya que están directamente relacionadas con el bienestar de docentes y estudiantes, lo cual, a su vez, es esencial para el aprendizaje. No obstante, además de la experticia pedagógica y de las competencias 
emocionales, existe otro atributo de igual relevancia para el personal docente universitario en el siglo XXI: lograr que sus estudiantes aprendan.

Atender a la totalidad de estudiantes según sus particularidades es esencial para cualquier educador en la actualidad. La mayoría se da por satisfecha cuando un número de sus estudiantes aprende. Sin embargo, un profesorado responsable procura que cada persona lo haga. Así lo afirma Bain (2007):

Reconocemos que algunos profesores pueden tener muchísimo éxito a la hora de ayudar a unos cuantos estudiantes a aprender, pero mucho menos cuando se trata de ayudar a la mayoría de ellos. (...) Nosotros buscamos personas que sí pueden conseguir peras de los que otros consideran que son olmos, personas que ayudan constantemente a sus estudiantes a llegar más lejos de lo que los demás confían. (p. 18)

Existe una manera de conseguir que todo el estudiantado aprenda y crezca: enseñar según la individualidad. Sabemos que cada persona es diferente y, por tanto, sus necesidades y destrezas son distintas también de los otros individuos. Esto significa que el personal docente debe asumir esas particularidades de sus estudiantes como base para diseñar sus procesos de aprendizaje. Como se comentará en los párrafos siguientes, existe evidencia científica reciente que demuestra la obligación que tiene la persona educadora de tomar en cuenta la individualidad de sus estudiantes.

Los avances tecnológicos desarrollados precisamente en el siglo XXI han permitido el crecimiento de campos de estudio cuyo análisis depende, justamente, del alcance de los artefactos que se utilizan para su investigación. Un claro ejemplo es la neurociencia. Gracias a estos avances tecnológicos se ha podido estudiar el cerebro a profundidad, lo que se ha traducido en descubrimientos de gran relevancia para la educación.

Uno de estos hallazgos tiene que ver con que no hay dos cerebros iguales, aunque comparten áreas con las mismas funciones. Tokuhama-Espinosa (2013) expresa:

Hay sin duda patrones generales del desarrollo del cerebro que todos compartimos, pero la singularidad de cada cerebro explica por qué los estudiantes aprenden de maneras sutilmente 
diferentes. Existe por otra parte un gran número de libros populares que tratan de explotar este hallazgo utilizándolo como excusa para explicar la imposibilidad de los maestros de llegar a todos los estudiantes. Esto es simplemente irresponsable. La singularidad de cada cerebro no tiene por qué ser eclipsado por el hecho de que los humanos como especies [sic] compartan etapas de desarrollo claras que marcan parámetros en el aprendizaje. (p. 19)

Este argumento es esencial para cualquier persona que se dedique a la educación. Como docentes, sabemos que cada persona aprende de formas diferentes y ahora hay evidencia científica que explica por qué: cada cerebro es único. Entonces, ¿por qué muchas veces pretendemos que todo nuestro estudiantado aprenda con las mismas experiencias de formación?, ¿por qué nos conformamos con que únicamente una parte lo haga? Hay que procurar que cada persona que asiste a nuestras clases viva experiencias positivas de aprendizaje y, para lograrlo, es necesario volver la mirada a la individualidad. No es posible educar sin tomar en cuenta al individuo, a quien este esfuerzo se dirige. Una formación estándar no puede atender a cada persona que la recibe porque es inflexible, no cambia con el tiempo, ni con las necesidades de la población que sí están en constante transformación.

Existe una frase que ejemplifica el gran error que cometemos al pretender que todas las personas se adapten a un sistema estandarizado. Dice que todos los seres somos genios, pero si juzgas un pez por su habilidad para trepar árboles, vivirá toda su vida pensando que es un inútil. Eso es precisamente lo que hacemos a nuestros y nuestras estudiantes cuando priorizamos el sistema por encima del individuo: les convertimos en peces intentando trepar árboles. Por lo tanto, tenemos que erradicar esa idea de que el sistema (para efectos de educación aplica también para experiencias de aprendizaje) es lo primero, por tanto, el individuo debe adaptarse a él y, si no lo hace, su destino es fracasar. Esta idea proviene de Taylor (1914), cuya propuesta de estandarización a nivel empresarial de principios del siglo XX provocó una transformación social importante (tanto que se mantiene hasta hoy) en múltiples áreas, entre ellas la educación (Rose, 2016).

El error que cometemos al pretender que la totalidad de estudiantes se adapten a un sistema o formación basada en lo estándar radica en que la manera que este se concibe y se estudia, no responde a las características 
del ser humano. De acuerdo con Rose (2016), la matemática de lo estándar se denomina estadística porque trata valores estáticos: estables, que no cambian. Sin embargo, sabemos que el ser humano sí cambia. Por ende, para entender de manera apropiada al individuo se necesita un tipo de matemática diferente, conocido como valores dinámicos: la matemática de valores cambiantes, no lineales, lo cual significa que lo estándar no funciona cuando de individuos se trata. Por eso, un sistema basado en la estandarización -y por ende en la estadística- no puede ser prioridad por encima del individuo. El estudiantado no puede ser tratado como estadística, como seres estáticos. El ser humano se caracteriza por su diversidad, individualidad y dinamicidad.

Es en sus estudiantes donde el sistema educativo encuentra su razón de ser. Nuestra prioridad deben ser siempre sus personas, no el sistema y, mucho menos, uno cuya característica principal es su rigidez. No se trata de bajar la calidad de la formación universitaria, sino de que esta misma realmente procure el crecimiento de todas de las personas que la reciben. Con ello, la calidad de la educación superior no tendría otro camino que mejorar. Se disminuirían en gran medida ese tipo de profesionales que, como se dice popularmente, fueron a la universidad, pero la universidad no pasó por ellos, pues a pesar de tener un título no demuestran haber aprendido. Educar desde la individualidad y hacia esta es una forma de asegurar el aprendizaje de la totalidad de estudiantes.

Por mucho tiempo hemos considerado que tenemos igualdad de oportunidades, cuando en realidad contamos con igualdad de acceso a las mismas experiencias, que además son limitadas porque fueron diseñadas como parte de un sistema estandarizado que no entiende de individualidades. Si el entorno no encaja con nuestra individualidad, nuestro desempeño no va a ser el mejor; mientras que si encajamos de manera positiva con nuestro entorno, tendremos la oportunidad de demostrar verdaderamente de lo que somos capaces (Rose, 2016).

Esta necesidad de atender las particularidades de cada persona queda también evidenciada por la población estudiantil, que agradece los esfuerzos por parte de sus docentes para dar una atención apropiada, en especial cuando se brindan ayudas o recomendaciones personalizadas. Esto lo afirma Giné (2009), quien expresa que "se valora, por parte del estudiantado, que el profesorado universitario pueda disponer de la posibilidad y tener la voluntad de proporcionar ayudas puntuales y personalizadas, en forma de propuestas particulares para alumnos específicos” (p. 131). 
Tan esencial resulta atender las necesidades individuales que, desde el momento en que descubrió que el estudio a partir del promedio y lo estándar es más que deficiente cuando se trata del individuo, Peter Molenaar fundó la Unidad de Metodología Cuantitativa de Sistemas en Desarrollo del Instituto de Investigación en Ciencias Sociales de Penn State University en el 2005. En esta institución, junto a un equipo científico, Molenaar empezó a crear las bases de lo que hoy se denomina la ciencia del individuo (Rose, 2016), la cual se ha ido desarrollando con el tiempo, también, en otras universidades prestigiosas. Tal es el caso de Harvard, que cuenta con el Laboratorio para la Ciencia del Individuo, donde se desarrollan nuevos conocimientos acerca de la individualidad, utilizados para abordar problemáticas educativas y sociales (The President and Fellows of Harvard College, 2018).

Todo esto, incluida la creación de nuevos campos de estudio para atender la individualidad, evidencia que es responsabilidad del profesorado universitario del siglo XXI tener una visión del aprendizaje desde lo individual, en la cual prime la atención adecuada de cada persona según sus particularidades, potenciándolas más y dejando de lado la idea de que todos y todas debemos ser lo más parecido posible al promedio, pero un poco mejor. Esto requiere mayor esfuerzo por parte de los grupos profesionales en educación, ya que significa un cambio radical en la forma de concebir el acto educativo y sus propósitos, así como diseñar y evaluar las experiencias de aprendizaje. Además, para lograrlo es necesario conocer bien a sus estudiantes, lo cual crea un vínculo más cercano entre docentes-estudiantes que, a su vez, exige y permite el desarrollo de las competencias emocionales mencionadas anteriormente.

A educar según la individualidad, a tener experticia pedagógica y a desarrollar competencias emocionales debemos agregar el dominio de herramientas tecnológicas, como otra de las características que requiere el personal docente universitario en la actualidad. Esto es porque parte de los rasgos distintivos de nuestra época es el desarrollo de la tecnología, que ha cambiado la forma en que vivimos por completo. Tenemos la posibilidad de conocer en tiempo real lo que sucede en cualquier parte del mundo a través de transmisiones en vivo en redes sociales y nos podemos comunicar por videollamadas, mensajes o correos electrónicos con personas de cualquier país del planeta.

Estas facilidades para la comunicación han tenido gran injerencia en los fenómenos de migración, ya que las empresas desarrollan proyectos 
a nivel internacional y las universidades ofrecen becas a personas de cualquier país. Según la Organización Internacional para las Migraciones (2011, según se cita en UNESCO, 2015b), "se puede estimar que uno de cada siete habitantes del planeta, lo que equivale a unos mil millones de personas, están 'ON THE MOVE' en el mundo de hoy" (p. 66).

Para la educación, la estadística mencionada significa que ya quedarse en lo nacional no es suficiente. Algo tan sencillo como la ciudadanía ya no puede verse como se hacía en el pasado, porque ahora implica personas de todas partes del mundo. La ciudadanía ya no se reduce al nacionalismo, sino que el concepto se ha transformado hacia ser ciudadanía del mundo. La educación para la ciudadanía mundial es impulsada por la UNESCO, forma parte del objetivo de desarrollo sostenible 4 de la agenda 2030, específicamente la meta 4.7, que afirma que se debe "asegurar que todos los alumnos adquieran los conocimientos teóricos y prácticos necesarios para promover el desarrollo sostenible, entre otras cosas mediante (...) la ciudadanía mundial" (UNESCO, 2017, p. 14), e implica inculcar "los valores, las actitudes y los comportamientos que constituyen la base de una ciudadanía mundial responsable: creatividad, innovación y compromiso a favor de la paz, derechos humanos y desarrollo sostenible" (UNESCO, 2018, párr. 3).

Ante esta realidad, el personal docente universitario -como actor responsable de la formación de personas- debe estar al tanto del bagaje de sus estudiantes y los acontecimientos más relevantes a nivel nacional e internacional, así como de las tendencias mundiales en su área de experticia, la educación, comunicación y avances científicos y tecnológicos que permiten la evolución de la sociedad.

Quien educa tiene el compromiso no solo de estar al tanto de todos estos avances, sino también de aprender a trabajar con base en ellos, siempre considerando que estas personas, a quienes forma, se deben desempeñar en esta coyuntura y responder a sus necesidades con criticidad, respeto, humanismo e innovación. No se puede dar una educación descontextualizada, y para lograrlo, las tecnologías de la información y comunicación, responsables en gran parte de la globalización, movilidad de personas y el auge de la ciudadanía mundial, resultan esenciales. Así se afirma en la Declaración de Quingdao (UNESCO, 2015a):

Los progresos considerables en relación con las TIC y la rápida expansión del acceso a Internet han convertido al mundo actual 
en un lugar cada vez más interconectado, y han hecho que conocer y estar familiarizado con las TIC sea esencial para cada niña y cada niño, y para cada mujer y cada hombre. (p. 21)

Es fundamental, entonces, trabajar en una alfabetización tecnológica crítica, que asegure que el estudiantado, que en su mayoría domina todas estas tecnologías de la información y la comunicación, las puedan utilizar de manera ética y analítica. En la actualidad, tienen la gran ventaja de poder acceder al conocimiento a través de internet. En menos de un segundo es posible encontrar miles de páginas web con información de todo tipo. En la educación, entonces, además de procurar la construcción de conocimientos por parte del estudiantado, debe ofrecer formación en temas como el uso ético de las TIC, analizar de forma crítica cada página web para discernir si su contenido es veraz y relevante, entre otros. Así lo manifiesta Area (2015, p. 23):

La alfabetización, desde esta perspectiva, debe representar la adquisición de las competencias intelectuales necesarias para interactuar tanto con la cultura existente como para recrearla de un modo crítico y emancipador y, en consecuencia, como un derecho y una necesidad de los ciudadanos de la sociedad informacional. Por todo ello, la escuela debiera ofrecer la igualdad de oportunidades en el acceso a los recursos tecnológicos y sobre todo en la capacitación para el uso inteligente y culto de la información y del saber. La multialfabetización de toda la ciudadanía es una condición necesaria para un desarrollo social armonioso y democrático de la sociedad del siglo XXI.

Así como el concepto de ciudadanía se transforma hacia lo mundial debido a los procesos migratorios derivados de la globalización, el desarrollo de TIC nos exige también pensar en una ciudadanía digital, que a su vez debe ser parte de la formación que el docente universitario ofrece. La ciudadanía digital abarca nuestra huella en entornos virtuales: implica cuestionarnos qué tan ético es nuestro uso de la tecnología, la cual ha llegado a revolucionar las leyes, al punto de poder tipificar como delitos acciones que anteriormente no imaginábamos.

La ciudadanía digital también conlleva aspectos relacionados con el ejercicio de derechos y deberes, esto porque la tecnología se ha 
convertido en una herramienta para el empoderamiento ciudadano. Hoy día las redes sociales, blogs y aplicaciones de mensajería son medios por los cuales se organizan movimientos ciudadanos (incluyendo algunos que pueden paralizar un país, como la huelga de 80 días en contra del Plan Fiscal en Costa Rica en el 2018), se hace control político a funcionarios públicos e incluso se desarrollan iniciativas sociales. Es por todo esto que, además de estar al tanto de los avances tecnológicos y trabajar en una alfabetización digital crítica, el personal docente universitario debe formar para una ciudadanía no solo física, sino también digital.

Nuestra sociedad está compuesta por personas de todas partes del mundo, con acceso inmediato a todo tipo de información. Más allá de estar desarrollando cada día más herramientas útiles en entornos de aprendizaje -que el personal docente, por supuesto, debe manejar-, vivimos en un mundo en que los avances tecnológicos han permeado en la forma en que interpretamos y hacemos leyes, interactuamos, producimos conocimiento, nos informamos, nos comunicamos y ejercemos nuestros derechos. Esto exige que el profesorado universitario esté empapado del desarrollo de la tecnología y sus implicaciones, ya que va a formar personas que necesitan desenvolverse en esta sociedad y deben hacerlo de manera crítica, por lo que es esencial que haga un uso pertinente y ético de las herramientas de las que dispone para ejercer su profesión.

Las condiciones descritas acerca de los fenómenos migratorios y las implicaciones que ha traído el desarrollo tecnológico a nivel político y social han tenido como consecuencia también la movilidad profesional. A nivel de la educación superior, esto ha provocado la creación de redes académicas en las cuales sus docentes trabajan en forma colaborativa, de manera que pueden realizar propuestas de importancia nacional y regional. Pertenecer a estas redes es otro aspecto para tener en cuenta por quienes ejercen la docencia universitaria en la actualidad.

Así como se valora el pertenecer a la ciudadanía del mundo debido a los fenómenos derivados de la migración y los avances de las TIC, se necesita también que el personal docente universitario trabaje en equipo. En conjunto con sus pares en el extranjero, deben procurar la mejora de la región, a través del acompañamiento en diferentes procesos que permitan a los grupos académicos beneficiarse de las experiencias de sus colegas a la hora de desarrollar proyectos. De acuerdo con Reynaga y Farfán (2004): 
[La importancia de las redes académicas] es enorme porque permite a los académicos trabajar con flexibilidad, cooperativamente, en el desarrollo académico, científico, técnico, social y cultural en una comunidad, equipo, grupo o región. Permite la integración para la solución de problemas y temáticas comunes, extiende beneficios a funcionarios, educadores, profesores, empresarios, sindicatos; puede constituirse por instituciones, secretarías, centros de investigación; facilita el intercambio de datos, información, conocimiento, y propicia la reflexión. Es un medio para crear fuentes de financiamiento y ofrece una herramienta a la comunidad. Tiene como fines intercambiar, construir, apoyar conocimientos, abonar a la solución de problemas, incrementar el número de investigadores, fortalecer posibilidades. (p. 8)

Estas redes brindan también un espacio para la producción, socialización e intercambio de conocimientos, además de ser un canal mediante el cual se puede gestionar la movilidad de docentes de universidades, que implica que hagan pasantías e investigaciones en universidades extranjeras, que crezcan como profesionales y que se empapen de las experiencias vividas en otros contextos.

Esto es particularmente importante considerando que una de las tendencias en la educación superior es la internacionalización, la cual busca que las universidades tengan estándares de calidad más altos al compararse con sus similares y respondan también a los fenómenos de movilidad estudiantil a través de regulaciones que faciliten, entre otros, procesos de equiparación. Es decir, si tanto las universidades como sus estudiantes valoran una visión y las experiencias internacionales, ¿cómo no vamos a esperar que el personal docente también lo haga?

Reynaga y Farfán (2004) son claros en cuanto a las formas en las que docentes de universidades pueden beneficiarse de pertenecer a redes académicas y acogerse a la movilidad laboral:

Hablar de compartir, intercambiar o difundir recursos, información, experiencias, resultados de práctica profesional, docente de investigación y difusión, son sólo algunos de los aspectos que permite el aprendizaje cooperativo entre académicos. Trabajar en una comunidad académica de conocimiento que mantiene una red, implica trabajar y aprender de manera cooperativa, abriendo 
espacios para conocer a otros académicos y sus vivencias, compartir objetivos y estrategias, facilitar la auto evaluación [sic] y la innovación, y valorar esfuerzos y trabajos ya hechos. Aprender entre académicos de manera común implica superar el enfoque de aislamiento por uno de cooperación, un cambio de actitud personal frente a los problemas, ampliar los horizontes, capacidades y potencialidades del uno por el uno y los otros. (p. 13)

Si la tecnología ha revolucionado la forma en que nos comunicamos y vemos el mundo, así como nuestro sentido de pertenencia internacional, es apenas lógico que el personal docente universitario se adapte a esas necesidades que se presentan en la actualidad y actúe conforme a ellas. Hacer comunidad internacional es fundamental, se ve en las iniciativas de organizaciones como la UNESCO y su agenda 2030, y el personal académico puede hacerlo a través de la conformación de redes y la movilidad.

Ahora bien, hacer comunidad internacional y ser ciudadanos y ciudadanas de mundo conllevan un reto más para la docencia universitaria en el siglo XXI: el dominio de un segundo idioma. Muchas de las actividades propias del quehacer docente se maximizan si este habla una lengua extranjera. La producción de nuevos conocimientos es de las características más importantes en la sociedad actual. Nuevas y múltiples investigaciones tienen lugar día tras día en todas partes del mundo. ¿Cómo pretende, entonces, la educadora y el educador universitario estar al día con las más recientes publicaciones, si es incapaz de entender gran parte de ellas por barreras lingüísticas?

La globalización, la movilidad profesional, la necesidad de hacer trabajo colaborativo con docentes de diversas partes del mundo, la posibilidad cada vez más común de realizar pasantías en el extranjero y participar en congresos en diferentes países, el ser ciudadanía del mundo y las implicaciones de vivir en un planeta cada vez más conectado a través del internet traen consigo la necesidad de comunicarse, de manera efectiva, en al menos una lengua extranjera.

Además de hablar un segundo idioma, otra característica esencial que este perfil docente universitario requiere en la actualidad es desarrollar procesos de reflexión y mejoramiento continuos. Puede empaparse de la realidad actual y hacer su mejor esfuerzo por responder a ella, según la naturaleza de los procesos de aprendizaje y las necesidades 
de sus estudiantes; pero si no procura su mejoramiento profesional a lo largo de la vida, llegará el momento en que se estanque y se convierta en un o una profesional desactualizada, con poca capacidad de enfrentarse a las necesidades del mundo que le rodea -que se transforma constantemente- presenta.

Por esto el personal docente del siglo XXI nunca deja de aprender, reflexiona sobre su praxis, la transforma y crece cada día como profesional. Para lograr esto, es responsable de enterarse de los más recientes avances, tanto en el área pedagógica como en su área de especialidad y otros campos relacionados (y no relacionados) con estas:

Los profesores extraordinarios están al día de los desarrollos intelectuales, científicos o artísticos de importancia en sus campos, razonan de forma valiosa y original en sus asignaturas, estudian con cuidado y en abundancia lo que otras personas hacen en sus disciplinas, leen a menudo muchas cosas de otros campos (en ocasiones muy distintas al propio) y ponen mucho interés en los asuntos generales de sus disciplinas. (Bain, 2007, p. 27)

Es de amplio conocimiento la importancia del aprendizaje a lo largo de la vida y de que la educación sea pertinente en el momento histórico en que se desarrolla (Delors, 1996). Es imperativo que el personal docente universitario tenga esto siempre presente en su quehacer y tome conciencia de que no aplica únicamente a sus estudiantes, sino también a su propia persona. Nunca deben dejar de aprender, lo cual implica indagar, leer, asistir a eventos de desarrollo profesional como cursos y congresos, estar al tanto de los últimos avances y tendencias en diversas áreas y sus implicaciones en la sociedad. De lo contrario, terminará brindando una educación descontextualizada que no responde a la coyuntura en la que vive.

Cabe destacar que, a pesar de la innegable importancia que tiene la actualización profesional, esta, por sí sola, resulta insuficiente para el mejoramiento continuo de un educador. Debe ir de la mano con la reflexión. Volver los ojos hacia lo que hemos hecho y analizar nuestro propio desempeño es un ejercicio de vital importancia en la profesión docente. Zabalza (2002) enfatiza cada docente debe reflexionar sobre su propia práctica, ya que "la práctica puede reforzar el hábito pero si no se analiza, si no se somete a contraste y no se reajusta podemos pasarnos la vida cometiendo los mismos errores" (p. 17). 
Es través de la reflexión sobre nuestra praxis y el enriquecimiento de nuestros saberes que podemos diseñar estrategias para transformarla, porque nos permite ver en qué estamos fallando, qué aspectos se pueden mejorar y cómo podemos incorporar nuevos conocimientos a nuestro quehacer. Es imperativo, entonces, que el personal docente universitario desarrolle un ciclo de praxis-reflexión-transformación que aplique, de forma permanente, de la mano con el desarrollo profesional.

\section{Reflexiones finales}

El siglo XXI ha tenido como cualidad distintiva la transformación vertiginosa y continua. El mundo en el que vivimos no es el mismo de hace unos años, este está caracterizado por los avances tecnológicos, la movilidad y la internacionalización, pero sobre todo por estar en constante cambio. Cambio que nunca deja de ser y que generalmente es mucho más rápido que la capacidad de nuestros sistemas e instituciones educativas para adaptarse a él. Hemos entendido que modificar nuestros sistemas tiempo después de que se presenta una necesidad y, aun así, conseguir que el cambio supla esas deficiencias antes de que ocurra otra transformación no funciona más, porque las alteraciones que se dan actualmente son demasiado rápidas para esto.

Ante esta clara insuficiencia para responder a la evolución tecnológica y social a tiempo, hemos optado por resaltar la importancia de la habilidad que tiene el ser humano para adaptarse. Buscamos profesionales y una ciudadanía que puedan ajustarse al cambio, que puedan responder rápidamente a las necesidades de un mundo que está en constante evolución. No podemos pretender que esto suceda, si quienes están a cargo de la educación, de formar a las personas que viven en este mundo de cambios vertiginosos, se quedan en el pasado; si no se adaptan primero, si consideran que los históricos requisitos de experticia disciplinar, experiencia profesional y producción académica son suficientes para ser un buen personal docente universitario en este contexto.

Una persona docente universitaria necesita mucho más que eso para poder hacer lo que se requiere en la actualidad. Necesita experticia pedagógica para comprender la naturaleza de los procesos de aprendizaje y con ello diseñar las experiencias educativas de manera apropiada. Es su responsabilidad manejar competencias emocionales para que las pueda utilizar y desarrollar en sus estudiantes, con el fin de que, en conjunto, ambos grupos se desempeñen de manera adecuada en un entorno 
caracterizado por procesos afectivos sumamente exigentes. Debe educar a partir de la individualidad para que logre atender a la totalidad de sus estudiantes y que cada estudiante pueda realmente dar su máximo potencial.

Le corresponde también dominar herramientas tecnológicas y sus implicaciones, de forma que procure una alfabetización tecnológica ética y crítica en la ciudadanía y futuros grupos profesionales del país. Es preciso que pertenezca a redes académicas que le permitan trabajar en forma colaborativa en proyectos de relevancia nacional y regional, acogerse a la movilidad laboral y tener una visión internacional de su quehacer para que pueda estar al tanto de las más recientes tendencias educativas y de su especialidad. Por último, resulta esencial que procure siempre la reflexión y el mejoramiento continuo con el propósito de enriquecer y transformar su praxis, según las particularidades de sus estudiantes y el contexto en que viven.

Si no es capaz de desarrollar estos atributos, al menos en su mayoría, se estanca y se aleja de la realidad, se encierra en un mundo diferente al que habita. La formación que puede ofrecer resulta deficiente porque no atiende las demandas de la sociedad, está desligada del escenario actual.

Una educación descontextualizada no puede ser pertinente y hoy más que nunca nuestra sociedad requiere una formación de calidad. El personal docente universitario del siglo XXI tiene en sus manos un reto al que debe hacer frente "con una actitud proactiva, con compromiso profesional, académico, ético y ciudadano" (Quintanilla, 2013, p. 4). No puede, entonces, ignorar aquello que le rodea, quedarse donde está, dejar de informarse, actuar siempre de la misma manera. Debe transformarse constantemente para poder atender las exigencias emergentes del acto educativo en un entorno cambiante y hacer su trabajo en forma adecuada. Su ética y responsabilidad profesional así lo demandan.

\section{Referencias}

Area, M. (2015). La alfabetización digital y la formación de la ciudadanía del siglo XXI. Revista Integra Educativa, 7(3), 21-33. http://www.scielo.org.bo/scielo.php?script=sci_arttext\&pid $=$ S1997-40432014000300002

Bain, K. (2007). Lo que hacen los mejores profesores de universidad (Traduc. Óscar Barberá). Palacios.

Bisquerra, R. (2011). Educación emocional: Propuestas para educadores y familias. Desclée de Brower. 
Bisquerra, R. (2016). 10 ideas clave: Educación emocional. Graó.

Delors, J. (1996). La educación encierra un tesoro: Informe a la UNESCO de la Comisión Internacional sobre la Educación para el siglo XXI. https://unesdoc.unesco.org/ark:/48223/pf0000109590_spa

Esteras, J., Sandín, B. y Chorot, P. (2016). El síndrome de burnout en los docentes: Los efectos de las variables psicosociales. Psicología y educación: presente y futuro. ACIPE. https://rua.ua.es/dspace/bitstream/10045/64387/1/Psicologia-y-educacion_138.pdf

Giné, N. (2009). Cómo mejorar la docencia universitaria: El punto de vista del estudiantado. Revista Complutense de Educación, 20(1), 117-134. http://revistas.ucm.es/index.php/RCED/article/ view/RCED0909120117A/15402

Goleman, D. (2015). Cómo ser un líder: ¿Por qué la inteligencia emocional sí importa? Nomos Impresores.

Mérida, S. y Extremera, N. (2017). Estado de la cuestión sobre inteligencia emocional y burnout en el profesorado por países, año de publicación, ciclos educativos e instrumentos de evaluación. Profesorado: Revista de curriculum y formación del profesorado, 21(3), 371-389. https://recyt.fecyt.es/index.php/profesorado/ article/viewFile/59803/pdf_53

Quintanilla, M. (2013). Una docencia pertinente para la sociedad del conocimiento. Revista UNAH INNOV@, 2, 4-5. https://lamjol. info/index.php/UNAHINNOV/article/download/2370/2137

Reynaga, S. y Farfán, P. (2004, febrero). Redes académicas...Potencialidades académicas. Cuarto Congreso Nacional y Tercero Internacional: "Retos y Expectativas de la Universidad". Conferencia llevada a cabo en el congreso ANUIES, México. http:/www. anuies.mx/media/docs/89_2_1_1012161230Reynaga_Obregon_y_Farfan_Flores_Redes_Academicas.pdf

Rose, T. (2016). The end of average: How we succeed in a world that values sameness. Harper One.

Taylor, F. (1914). The principles of scientific management. Harper \& Brothers. https://play.google.com/books/reader?id=loU0AQAAMAAJ\&hl=es\&pg=GBS.PA3

Tecnológico de Costa Rica. (2018a). Reglamento del régimen de enseñanza-aprendizaje del Instituto Tecnológico de Costa Rica y sus reformas. https://www.tec.ac.cr/reglamentos/reglamento-regimen-ensenanza-aprendizaje-tecnologico-costa-rica-sus-reformas 
Tecnológico de Costa Rica. (2018b). Reglamento de carrera profesional del Instituto Tecnológico de Costa Rica y sus reformas. https://www.tec.ac.cr/reglamentos/reglamento-carrera-profesional-instituto-tecnologico-costa-rica-sus-reformas

The President and Fellows of Harvard College. (2018). Laboratory for the Science of Individuality at the Harvard Graduate School of Education. https://lsi.gse.harvard.edu/

Tokuhama-Espinosa, T. (2013). ¿Qué puede hacer la ciencia de mente, cerebro y educación (MCE) por la enseñanza y el aprendizaje? Para el Aula, 5, 18-21. http://www.usfq.edu.ec/ publicaciones/para_el_aula/Documents/para_el_aula_05/0010_ para_el_aula_05.pdf

UNESCO. (2015a). Declaración de Quingdao: Aprovechar las oportunidades digitales. Liderar la transformación de la educación. http://unesdoc.unesco.org/images/0023/002333/233352m.pdf

UNESCO. (2015b). Replantear la educación: ¿Hacia un bien común mundial? http://unesdoc.unesco.org/images/0023/002326/232697s.pdf

UNESCO. (2017). Desglosar el objetivo de desarrollo sostenible 4: Educación 2030. http://unesdoc.unesco.org/images/0024/002463/246300S.pdf

UNESCO. (2018). Educación para la ciudadanía mundial. https:// es.unesco.org/themes/ecm

Universidad de Costa Rica. (2008). Reglamento de régimen académico $y$ servicio docente. http://cu.ucr.ac.cr/normativ/regimen_academico_docente.pdf

Universidad Estatal a Distancia. (2018a). Estatuto de personal. https:// www.uned.ac.cr/academica/images/Normativa/Estatuto $\% 20$ de\%20personal.pdf

Universidad Estatal a Distancia. (2018b). Reglamento de carrera universitaria. https://www.uned.ac.cr/academica/images/cidreb/reglamento/admin-recursos-humanos/Carrera_Universitaria_18_ enero-2018.pdf

Universidad Nacional. (2015). Reglamento del régimen de carrera académica. http://documentos.una.ac.cr/handle/unadocs/8524

Universidad Nacional. (2018). Reglamento de contratación laboral para el personal académico de la Universidad Nacional. http:// documentos.una.ac.cr/handle/unadocs/5552 
Universidad Técnica Nacional. (2017). Manual descriptivo de clases de puestos. http://www.utn.ac.cr/sites/default/files/attachments/Manual $\% 20$ Descriptivo $\% 20 \mathrm{de} \% 20$ Clases $\% 20 \mathrm{de} \% 20$ Puestos $\% 20$ UTN\%20Reforma\%20III.pdf

Zabalza, M. (2002). La enseñanza universitaria. El escenario y sus protagonistas. Narcea.

Zabalza, M. (2009). Ser profesor universitario hoy. La Cuestión Universitaria, 5,68-80.http://polired.upm.es/index.php/lacuestionuniversitaria/ article/view/3338 
